\title{
ARTICLE
}

\section{The radiation assessment for the maintenance scenarios of CSNS inner reflector plug}

\author{
Quan-zhi $\mathrm{Yu}^{\mathrm{a} *}$, Zhi-liang Hu ${ }^{\mathrm{b}}$, Bin Zhou ${ }^{\mathrm{b}}$, Jian-fei Tong ${ }^{\mathrm{b}}$, You-lian Lu ${ }^{\mathrm{b}}$, \\ Wen Yin ${ }^{\mathrm{a}}$ and Tian-jiao Liang ${ }^{\mathrm{a}}$ \\ ${ }^{a}$ Beijing National Laboratory for Condensed Matter, Institute of Physics, Chinese Academy of Sciences, Beijing 100190, China; \\ ${ }^{b}$ Institute of High Energy Physics, Chinese Academy of Sciences, Beijing 100049, China
}

\begin{abstract}
China Spallation Neutron Source (CSNS) is a neutron facility with $100 \mathrm{~kW}$ beam power, which can be upgraded to $500 \mathrm{~kW}$ after several years irradiation. The moderator-reflector plug is divided as Inner Reflector Plug (IRP) and Out Reflector Plug (ORP) for cost saving. The IRP consists two moderators, beryllium (Be) reflectors and upper shielding blocks. It should be periodically replaced due to radiation damage caused by intensity neutrons and other secondary particles. Since the IRP is highly activated after several year irradiation, the intensity residual gamma rays result the severe challenge for its replacements and potential radiation exposure to the personnel. Detailed calculations about the radionuclides, activity, decay gamma rays and dose rate distributions of the IRP are performed. The dose rates to the personnel during the maintenance scenarios are assessed. The shielding casks used to transport these activated components are also designed. Detailed information about the dose rate distributions during the IRP replacement is analyzed. Calculations are performed by Monte Carlo code MCNPX2.5 and the activation code CINDER'90 combined with an Activation Script and a Gamma Source Script.
\end{abstract}

Keywords: China Spallation Neutron Source; inner reflector plug; maintenance scenarios; Monte Carlo; activity; dose rate; shielding cask

\section{Introduction}

China Spallation Neutron Source (CSNS) is a new facility being in construction in south of China [1]. It consists an accelerator delivering a $1.6 \mathrm{GeV}$ proton beam with $100 \mathrm{KW}$ beam power at a $25 \mathrm{~Hz}$ frequency on to a W-Ta target. Three different moderators are selected to slowdown the fast neutrons, supplying thermal neutrons to 19 instruments for scientific research. A high energy neutron beam is specially designed in the forward direction for single event effect (SEE) research. Beryllium (Be) and Iron (Fe) reflectors are used to increase thermal neutron intensity. Considering higher power neutron facilities built in the world, CSNS will be upgraded to $500 \mathrm{~kW}$ beam power after several years operation.

The moderator-reflector plug is separated into inner reflector plug (IRP) and outer reflector plug (ORP) for cost saving consideration. The decoupled water moderator (DWM) and the decoupled and poisoned hydrogen moderator (DPHM), which have life time about six years at $500 \mathrm{~kW}$ beam power, are fixed in the IRP section. While the coupled hydrogen moderator (CHM) with longer life time, is fixed in the ORP section. Radiation damage of structure materials exposed to high intensity neutrons and other secondary particles forces

*Corresponding author. Email: qzhyu@aphy.iphy.ac.cn periodic replacement of these moderators. Since these moderators and the surrounding components are highly activated and will emit high intensity residual gamma rays, severe challenge exists for their replacements and potential radiation exposure to the personnel.

In this paper, the radiation assessment of the IRP is much of concerned. Detailed information about the radionuclides, activity, decay gamma rays, dose rate distributions is calculated, as well as the thickness of the shielding casks. Calculations are performed by Monte Carlo code MCNPX2.5 [2] and activation code CINDER'90 [3] combined with an Activation Script and a Gamma Source Script, with variance reduction methods for decreasing the statistical errors.

\section{Codes and methods}

The calculation sequence of the residual gamma dose rate in the IRP is as follows. Firstly, MCNPX2.5 is used to simulate the proton beam interacting with the target station. Bertini intranuclear cascade model coupled with RAL evaporation-fission model is used to determine the spallation products for high energy interactions $(\mathrm{E}>20 \mathrm{MeV})$. The nuclear data library ENDF-B/VI is used to calculate the low energy neutron $(\mathrm{E}<20 \mathrm{MeV})$ fluxes in a 63 energy group structure. Secondly, the activation code CINDER'90 is used to determine the 
residual gamma source in the IRP at interested time steps. This code solves the Bateman equations and deduces the time dependence of isotope buildup and decay. Thirdly, an Activation Script provides the interface between MCNPX2.5 and CINDER'90, providing residual gamma rays of interested cells. And a Gamma Source Script extracts the gamma ray information as a new source file for different situations. Lastly, MCNPX2.5/MCNP4C is used to transport these gamma rays, for dose rate and shielding cask calculations.

All calculations are based on a $1.6 \mathrm{GeV}, 500 \mathrm{~kW}$ $\left(1.95 \times 10^{15} \mathrm{p} / \mathrm{s}\right)$ proton beam for 6 years irradiation (5000 hours full power operating time per year). The footprint proton beam profile is $16 \mathrm{~cm}$ width and $6 \mathrm{~cm}$ high, with double Gaussian distributions of $9.42 \mathrm{~cm}$ and $3.53 \mathrm{~cm}$ full width at half maximum (FWHM) in $\mathrm{x}$ and $\mathrm{z}$ directions. The cooling time for IRP maintenance is 7 days after the proton beam shut down. The acceptable dose rate is $1 \mathrm{mSv} / \mathrm{h}$ during IRP maintenance.

\section{Calculation results}

\subsection{Calculation model}

The tungsten target and the moderators were once detailed described at elsewhere [4]. The geometry model of the IRP is shown in Figure 1. It is divided with three sections along the height direction. The inner bottom (IB) section is above the tungsten target, with height of 130 $\mathrm{cm}$. It consists of the DWM, DPHM, Be reflector and a shielding block. The inner middle (IM) section is 110 $\mathrm{cm}$ high, including a shielding block with several pipes supplying for hydrogen and cooling water. The inner upper (IU) section, with height of $140 \mathrm{~cm}$, is also a shielding block but with a step structure. Except the moderators and reflectors, the IRP is composed with stainless steel (SS316) at present, with density of 7.8 $\mathrm{g} / \mathrm{cm}^{3}$. The composition are Fe57, 1.3\%, Cr52, 13.8\%, Cr53, 1.6\%, Ni58, 7.9\%, Ni60,2.4\%, Mn55, 1.9\%, in $\mathrm{g} / \mathrm{cm}^{3}$. The composition are Fe57, 1.3\%, Cr52, 13.8\%,

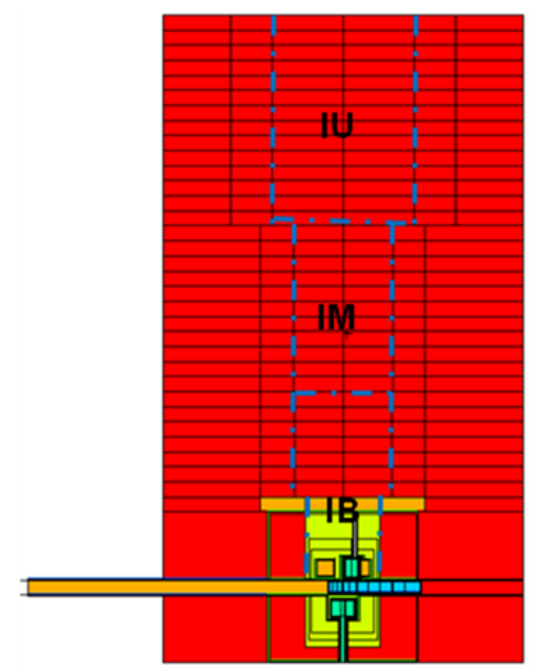

Figure 1 . The calculation model of the IRP. The height of the IB, IM and IU sections are $130 \mathrm{~cm}, 110 \mathrm{~cm}$ and $140 \mathrm{~cm}$.
Cr53, 1.6\%, Ni58, 7.9\%, Ni60,2.4\%, Mn55, 1.9\%, in mass density. After several years irradiation, the two decoupled moderators will reach their life time. The IU, IM and IB sections will be changed out accordingly at the vertical direction. The division of the IRP into three parts is according to the assessments of the neutronics performance, the thermal hydraulic analysis and the shielding.

\subsection{Activity and residual gamma ray}

The main radionuclides produced in the IRP are H3, Cr51, Mn54, Fe55, Co57, Co58, and Co60, which have more than $1 \%$ contributions to the total activity and have more than $1 \mathrm{MeV}$ high energy residual gamma rays. For better understanding of activity distributions in the IRP, the activity density at the centre position along the IRP is calculated, as shown in Figure 2. One can see that for $500 \mathrm{~kW}$ beam power, the activity density at $60 \mathrm{~cm}$ above the target high reaches $10^{8} \mathrm{~Bq} / \mathrm{cm}^{3}$. At $350 \mathrm{~cm}$ high, it is about $1 \mathrm{~Bq} / \mathrm{cm}^{3}$. The activity density presents an exponential decrease along the height of IRP. The activity density provides the initial information about the dose rate distributions in the three sections of the IRP. One can estimates that at $230 \mathrm{~cm}$, gamma dose rate is less than $1 \mathrm{mSv} / \mathrm{h}$, according the estimation of Refs. $[5,6]$. This implies that at $230 \mathrm{~cm}$ or higher position, the steel block can be pulled out without temporally shielding cask.

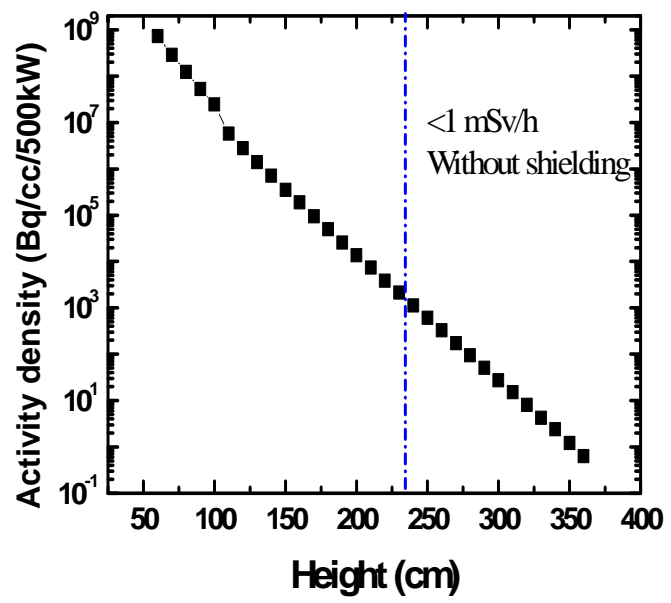

Figure 2. Activity density at the centre of the IRP after 7 days cooling. The dose rate is estimated less than $1 \mathrm{mSv} / \mathrm{h}$ at $230 \mathrm{~cm}$ above the target.

The activity in the IRP is also calculated and compared, among the IB, IM and IU sections. The activity evolution with decay time is showed in Figure 3. One can see that the largest activity appears in the IB section, which is nearer to the spallation target than the IM and IU sections. The total numbers of emitted photons from these three sections are also presented in Figure 3. It can be seen that the evolutions of the emitted photon numbers in the IB, IM and IU sections are very similar with that of the activity evolutions. The comparisons of the activity and the emitted photon 
numbers indicate that the main decay style from the radionuclides in the IRP is residual gamma ray, taking about $70 \%$ for most cases.

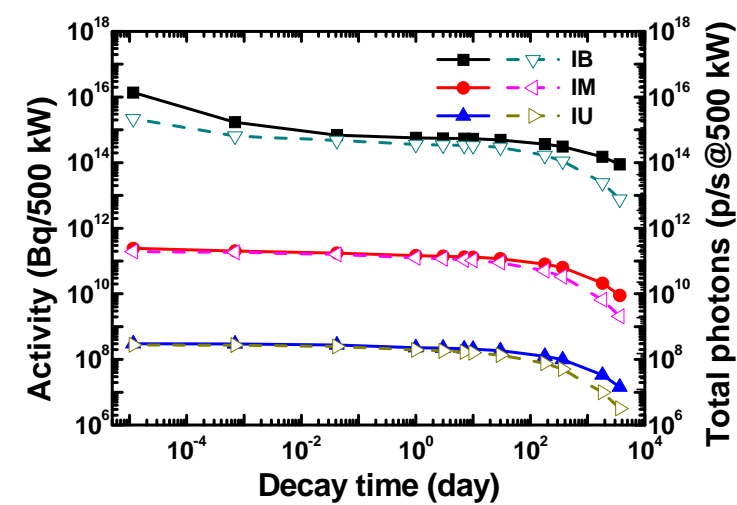

Figure 3. The evolutions of the activity and the total number of emitted photons in the IB, IM and IU sections. Solid symbols correspond to activity (left ordinate) and open symbols correspond to gamma intensity (right ordinate).

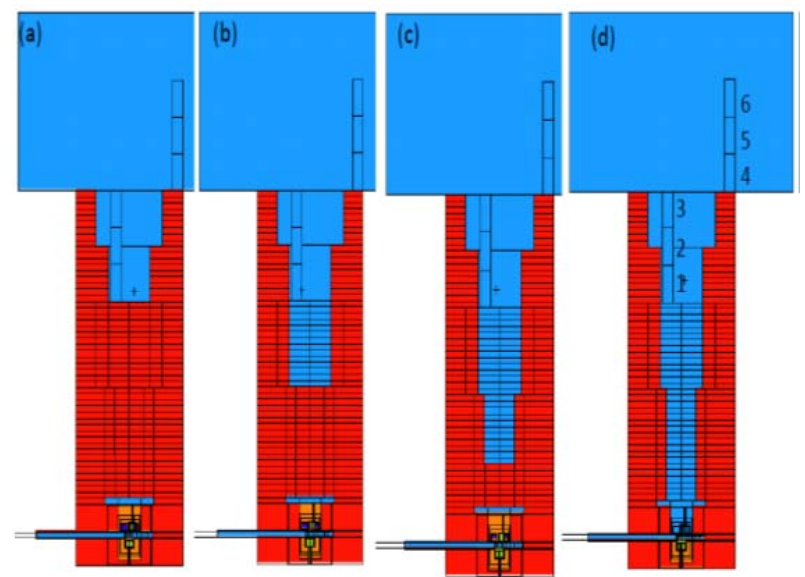

Figure 4. The maintenance scenarios of the IRP. (a) Top shielding block, (b) IU, (c) IM and (d) IB. 1 3 and 4 6 model the workers exposed in the radiation field during IRP maintenance.

\subsection{Dose rate distribution}

Figure 4 (a), (b), (c) and (d) show the four steps for the IRP maintenance scenarios, respectively. It means that for the replacements of the DWM and DPHM, the top shielding blocks should be pulled out firstly. Then the IU, IM and IB will be pulled out accordingly. 1 3 are three equal-volume cells, each with $12.5 \mathrm{~cm}$ radius and $60 \mathrm{~cm}$ long. They are used as detectors to simulate

Table 1. Dose rates in detectors corresponding to Figure 4(b).

\begin{tabular}{|c|c|c|}
\hline Detector & $\begin{array}{c}\text { Dose rate } \\
(\mu \mathrm{Sv} / \mathrm{h})\end{array}$ & $\begin{array}{c}\text { Calculation } \\
\text { error }\end{array}$ \\
\hline 1 & 10.7 & 0.014 \\
2 & 5.6 & 0.024 \\
3 & 3.1 & 0.036 \\
\hline 4 & 0.08 & 0.161 \\
5 & 0.15 & 0.114 \\
6 & 0.09 & 0.093 \\
\hline
\end{tabular}

the dose rate to the maintenance operator on top of the IRP, at $340 \mathrm{~cm}$ above the target.While $4 \sim 6$ are detectors for maintenance operator at $520 \mathrm{~cm}$ above the target. Calculation shows that when the top shielding block is pulled out, as shown in Figure 4 (a), the dose rate to the operators is far below $1 \mathrm{mSv} / \mathrm{h}$. It implies that the residual gamma rays from the IRP are greatly self shielded. While for the other three situations, the high dose rates should give much of concern. The dose rates corresponding to Figure 4(b) (c) and (d) are listed in Table 1, 2, and 3 respectively. The statistical errors are also listed for these detectors. One can see that the dose rate in Table 3 correspond to the worst situation during the IRP replacement. The reason is that without the shielding of the IM section, the intense gamma rays produced in the Fe reflectors can directly affect the operators during the IRP maintenance.

Table 2. Dose rates in detectors corresponding to Figure 4 (c).

\begin{tabular}{|c|c|c|}
\hline Detector & $\begin{array}{c}\text { Dose rate } \\
(\mu \mathrm{Sv} / \mathrm{h})\end{array}$ & $\begin{array}{c}\text { Calculation } \\
\text { error }\end{array}$ \\
\hline 1 & 213366 & 0.013 \\
2 & 131619 & 0.015 \\
3 & 84607 & 0.018 \\
\hline 4 & 297 & 0.053 \\
5 & 682 & 0.048 \\
6 & 1732 & 0.043 \\
\hline
\end{tabular}

Table 3. Dose rates in detectors corresponding to Figure 4 (d).

\begin{tabular}{|l|l|l|}
\hline Detector & $\begin{array}{l}\text { Dose rate } \\
(\mu \mathrm{Sv} / \mathrm{h})\end{array}$ & $\begin{array}{l}\text { Calculation } \\
\text { error }\end{array}$ \\
\hline 1 & 4136 & 0.023 \\
2 & 2712 & 0.031 \\
3 & 1842 & 0.039 \\
\hline 4 & 2.6 & 0.152 \\
5 & 17.7 & 0.075 \\
6 & 50.1 & 0.052 \\
\hline
\end{tabular}

\subsection{Shielding cask}

Since the IRP is highly activated, it should be extracted into the temporary shielding casks during its maintenance. For the IU section, as we estimated in section 3.2, no cask is needed during its replacement. While for the IM and IB sections, different thickness of lead $(\mathrm{Pb})$ casks are needed to shield the residual gamma rays. The radius of the IM section is $32.5 \mathrm{~cm}, 110 \mathrm{~cm}$ high. The Pb cask is out of the IM section, each cell with $2 \mathrm{~cm}$ thickness, as seen in Figure 5. Calculation shows that for the IM section, the thickness of the $\mathrm{Pb}$ cask is 6 $\mathrm{cm}$ in radius, and $6 \mathrm{~cm}$ at the bottom. For the IB section, however, the $\mathrm{Pb}$ thickness in radius is $10 \mathrm{~cm}$, the bottom lid is $10 \mathrm{~cm}$, and the top lid is $7.0 \mathrm{~cm}$. Such kind of shielding cask can temporally restore the IRP sections during it maintenance. The dose rate distributions for the IM and IB sections, with their geometries, are shown in Figure 5 and Figure 6, respectively.

Figure 7 shows the dose rate distribution along the radius $(\mathrm{r}=32.5 \mathrm{~cm})$ of $\mathrm{IB}$ section and the $\mathrm{Pb}$ cask. One 


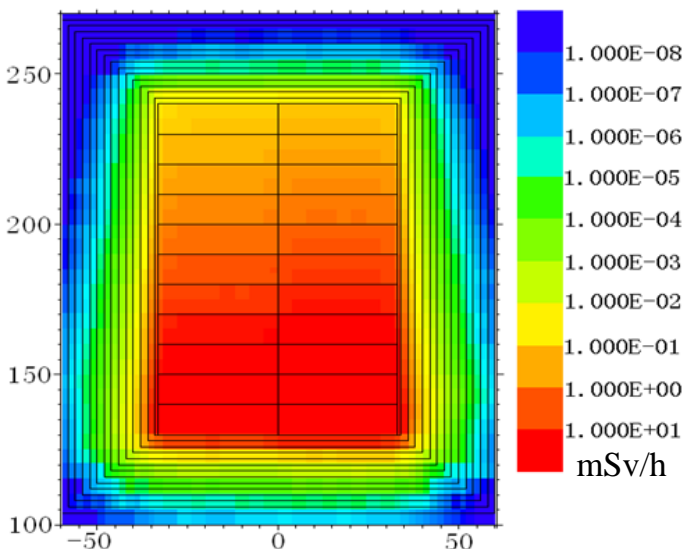

Figure 5. Distributions of gamma dose rate for the shielding of the IM section. $6 \mathrm{~cm}$ thickness of Pb cask is needed.

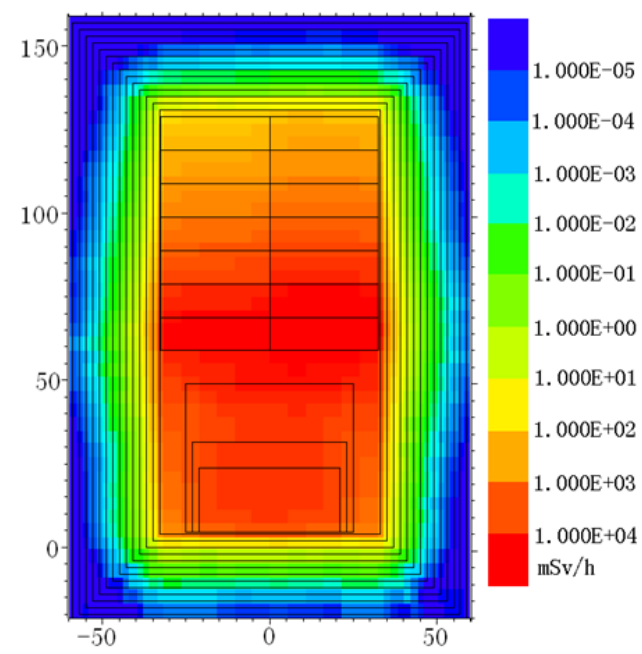

Figure 6. Gamma dose rate map for shielding the IB section. $\mathrm{Pb}$ cask with $10 \mathrm{~cm}$ in radius, $10 \mathrm{~cm}$ at the bottom and $7 \mathrm{~cm}$ at the top side is needed to decrease the dose rate $<1 \mathrm{mSv} / \mathrm{h}$.

can see that for the IB itself, the dose rate presents somewhat increase. It implies that in the forward direction of the proton beam, the residual gamma ray produced in the forward direction is higher than that in the centre of target station. The Pb layer can effectively decrease the dose rate exponentially. $10 \mathrm{~cm}$ thickness of $\mathrm{Pb}$ cask can decrease the dose rate less than $1 \mathrm{mSv} / \mathrm{h}$, which can be acceptable during its maintenance. Other materials of shielding casks are also in consideration.

\section{Conclusion}

During the design of CSNS target station, the life time of DPHM and DWM is found shorter than that of CHM. For cost saving consideration, the moderator-reflector plug is divided into IRP and ORP. These components are highly activated and release high intensity gamma rays, resulting the cautious operation during the maintenance procedure. Calculations and analyses were performed to assess the dose rates for the steps of the maintenance scenarios of the IRP. Calculation results showed that the activation along the centre of the IRP is decreased linearly. At $230 \mathrm{~cm}$ above the target, no cask

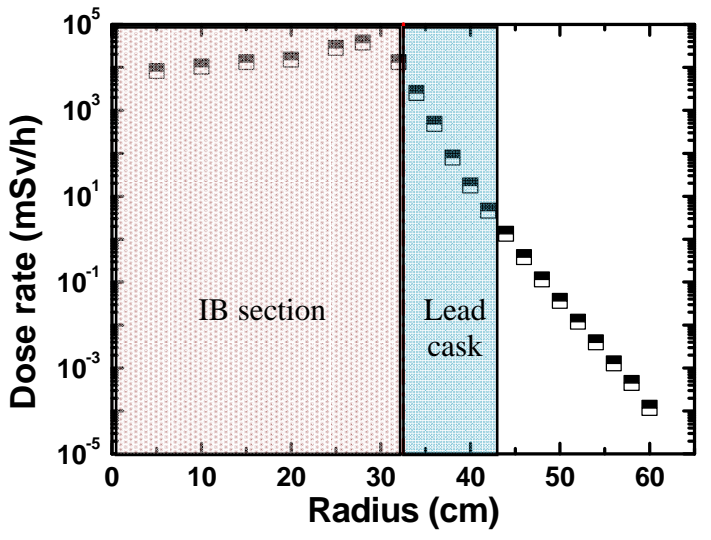

Figure 7. The radial dose rate distribution of IB section. The radius of the IB is $32.5 \mathrm{~cm} .10 \mathrm{~cm}$ thickness of heavy concrete can satisfy the shielding demand.

is required for the change out of the shielding block. While for the IM section, the thickness of Pb cask is about $6 \mathrm{~cm}$ in radius. For the IB section, $10 \mathrm{~cm}$ thickness of $\mathrm{Pb}$ cask is required. During the change out of each section of the IRP, the gamma dose rates to the operators at $340 \mathrm{~cm}$ and $520 \mathrm{~cm}$ above the IRP were assessed. These results provide great references for the IRP maintenance. All calculations are performed by the codes MCNPX2.5 and CINDER'90 and two important perl scripts.

Calculations of dose rate and shielding cask require repeatedly interactions with mechanical engineers for the actual models, such as the gaps, pipes, materials, void space, such kinds of calculations and design are going on.

\section{Acknowledgements}

The authors wish to thank Franz Gallmeier and Wei Lu (at SNS in ORNL) for fruitful discussion. This work was supported by the National Science Foundation of China (Grant Nos. 11075203, 91026009 and 11174358).

\section{References}

[1] http://csns.ihep.ac.cn

[2] MCNP/MCNPX CCC-730, Monte Carlo N-Particle Transport Code System Including MCNPX 2.5.0 and Data libraries, (2006).

[3] W. B. Wilson, T. R. England and K. A. Van Riper, Status of CINDER'90 Codes and Dat, LA-UR-99-316, Los Alamos National Laboratory, (1999).

[4] Q. Z. Yu, T. J. Liang and W. Yin, Activity and radiation protection studies for the $\mathrm{W}$-Ta target of CSNS, Radiat. Prot. Dosim. 136, 216 (2009).

[5] P. J. Gollon, Production of radioactivity by particle accelerators, IEEE Trans. Nucl. Sci. NS-23:1395 (1976).

[6] G. R. Stevenson, Induced activity in accelerator structures, air and water, Radiat. Prot. Dosim. 96, 373 (2001). 